\title{
Diabetes y enfermedad periodontal, una relación de doble vía
}

Diabetes and periodontal disease,

a two-way relationship
Diabetes e doença periodontal, uma relacion de dupla via
Fecha de Recepción

15 de agosto de 2017
Aceptado para su publicación

12 de julio de 2018
Profesor Titular. Asignatura Periodoncia. Módulos Patología y Diagnóstico I, II y III.

Email: jmonzon@odn.unne.edu.ar

Carlos Caramello Auxiliar Docente. Asignatura Periodoncia. Módulos Patología y Diagnóstico I, II y III.

Email: crcaramello@odn.unne.edu.ar

Miguel Jorge Acuña Jefe de Trabajos Prácticos. Asignatura Física Química Biológica. Módulo Morfofunción II. Email: mjacuna@odn.unne.edu.ar

Facultad de Odontología. Universidad Nacional del Nordeste.

Avenida Libertad 5450. (3400) Corrientes, Argentina. Tel: $+5493794457992 / 93$.

\section{Resumen}

La asociación entre diabetes mellitus y la enfermedad periodontal ha sido motivo de estudio durante mucho tiempo. Son varias las hipótesis que se barajan a la hora de explicar dicha relación. El propósito de este artículo, es revisar los estudios publicados en la literatura periodontal hasta la fecha sobre la relación entre enfermedad periodontal y diabetes mellitus.

\section{Palabras clave}

Diabetes mellitus, hiperglucemia, periodontitis.

\section{Summary}

The association between diabetes mellitus and periodontal disease has been studied for a long time. There are several hypothesis which have tried to explain this relationship. The purpose of this article is to review the studies published in the periodontal literature to date on the relationship between periodontal disease and diabetes.

Key words

Diabetes mellitus, hiperglycemia, periodontitis.

\section{Resumo}

A associação entre diabetes mellitus e a doença periodontal tem sido motivo de estudo durante muito tempo. São várias as hipóteses que se baralham à hora de explicar dita relação. O propó- 
DIVULGACIÓN

Diabetes y enfermedad periodontal, una relación de doble vía sito deste artigo é revisar os estudos publicados na literatura periodontal até o presente, sobre a relação entre diabetes e doença periodontal.

\section{Palavras chave}

Diabetes mellitus, hiperglicemia, periodontite.

\section{Introducción}

Los problemas periodontales pueden complicar el control de la diabetes, y una diabetes sin control puede agravar la enfermedad periodontal. Los estudios indican que gran parte de la población mundial tiene algún tipo de enfermedad periodontal incluyendo la forma más común, la periodontitis crónica del adulto. La diabetes y las enfermedades periodontales son desórdenes crónicos comunes en muchas partes del mundo. Una relación bidireccional es aquella en la que se reconoce que la diabetes afectaría adversamente la salud periodontal y la infección periodontal, por su parte, afectaría a la diabetes contribuyendo a un mal control glucémico y aumentando el riesgo de las complicaciones propias de ésta enfermedad metabólica'.

Debido a que la prevalencia tanto de la periodontitis crónica como de la diabetes se incrementa con la edad, establecer una relación entre ambas enfermedades en grupos de avanzada edad es dificultoso. Recientes estudios, en donde la relación de la edad con la enfermedad periodontal es tenida en cuenta, muestran que en personas con diabetes mellitus no dependientes de insulina (DMNDI), también llamada diabetes de tipo 2, la enfermedad periodontal es más severa y más prevalente que en personas sanas ${ }^{2}$.

La pérdida de tejido óseo, medida en milimetros alrededor de la raíz del diente, es un indicador clave de la severidad de la enfermedad periodontal. Se sabe que los indios Pima, de Arizona, constituyen la población con mayor prevalencia de diabetes tipo 2 en el mundo, $y$ en ellos la infección periodontal y pérdida de dientes son complicaciones significativas de la enfermedad. Dichos estudios muestran además que los adultos con diabetes tienen una mayor pérdida de dientes por enfermedad periodontal que las personas de edad similar que no han tenido diabetes según Taylor y Wornakke3.
La diabetes y la enfermedad periodontal también están presentes en niños y adolescentes. La gingivitis es más frecuente y severa en niños prepúberes con diabetes, especialmente cuando el control metabólico es pobre. Entre los adolescentes, aproximadamente el $16 \%$ de los niños entre los 8 y II años con diabetes mellitus tiene periodontitis 4

Estudios tradicionales a finales de los años 60 demostraron que los jóvenes adultos insulinodependientes mostraron un incremento significativo en la destrucción de los tejidos periodontales comparado con personas sin diabetes. Estos estudios mostraron además que individuos con diabetes del tipo I con cambios retinales experimentaron una mayor pérdida de soporte periodontal que aquellos sin esta complicación ocular ${ }^{5}$.

Como se esperaría, los reportes en la literatura proporcionan respuestas a diferentes preguntas y varían en su capacidad de ayudar a entender cómo la diabetes puede considerarse una causa de mala salud periodontal y a establecer qué tan fuerte es la relación. La mayoría de los reportes que brindan alguna información sobre el vínculo entre la diabetes y la prevalencia de la enfermedad periodontal provienen de estudios transversales, aunque un grupo más pequeño de estudios longitudinales y basados en la población también proporcionan soporte adicional para la asociación entre diabetes y enfermedad periodontal. Precisamente, los estudios de prevalencia permiten comparar las diferencias en porcentajes o proporciones de individuos con enfermedad periodontal entre aquellos con y sin diabetes. La gran mayoría de todos éstos estudios que hemos consultado concluyen en que la prevalencia es siempre mayor en personas que padecen diabetes.

Entonces es importante no solo para los profesionales de la odontología sino para todos los profesionales de la salud entender el papel y la importancia de la salud oral en el manejo de los pacientes con diabetes.

\section{Desarrollo}

Las enfermedades periodontales se asocian a un peor control de la glucemia en diabéticos. Las enfermedades periodontales también pueden influir en la diabetes. En un estudio clínico con una 
REVISTA FACULTAD DE ODONTOLOGÍA ISSN No 1668-7280 - Vol. XI No $1-2018$ 36

DIVULGACION

Monzón - Caramello - Acuña

duración de 2 años, se observó que había un peor control de los valores de glucemia en pacientes con diabetes tipo 2 en relación con los pacientes diabéticos tipo II sin enfermedad periodontal ${ }^{6}$. La diabetes es más frecuente en individuos con enfermedades periodontales. Se ha observado que los pacientes con enfermedades periodontales podrían tener diabetes tipo 2 con más frecuencia que los individuos con las encías sanas $^{7}$. El tratamiento periodontal podría mejorar el control de la glucemia en pacientes diabéticos. Un meta-análisis de 10 ensayos clínicos con más de 450 pacientes, encontró una disminución absoluta en los valores de hemoglobina glicosilada (HBAlc) del $0.4 \%$, después de realizar raspado y alisado radicular; si, además se añadía el uso de antibióticos sistémicos al raspado y alisado radicular, el resultado era una reducción del HBAlc del $0.7 \%{ }^{8}$. Aunque ninguna de esas reducciones era estadísticamente significativa, en la práctica de la medicina una reducción del $0.7 \%$ del $\mathrm{HbAl}$ c, es considerada clínicamente significativa ${ }^{8-9}$. Más recientemente, otro meta-análisis, en el que se revisaron 5 estudios con 379 pacientes, concluyó que el tratamiento periodontal permite una mejora de los niveles de glucemia de $0.40 \%$ en pacientes diabéticos tipo 2, durante al menos 3 meses 9 . Las enfermedades periodontales aumentan el riesgo de sufrir las complicaciones asociadas a la diabetes. Preshaw y col. han constatado los riesgos de sufrir periodontitis y diabetes conjuntamente: en diabéticos con periodontitis, la incidencia de microalbuminuria es 2 veces mayor, y la enfermedad renal terminal es 5 veces mayor, que en los diabéticos sin periodontitis; los diabéticos con periodontitis avanzada tienen 3 veces más riesgo de mortalidad cardiorenal (enfermedad cardíaca isquémica y nefropatía diabética, combinadas) que los diabéticos sin periodontitis ${ }^{10}$. Las enfermedades periodontales se asocian a un peor control de la glucemia en diabéticos. Los diabéticos con periodontitis avanzada tienen 3 veces más riesgo de mortalidad cardiorenal (enfermedad cardíaca isquémica y nefropatía diabética, combinadas) que los diabéticos sin periodontitis ¿Qué problemas de las encías puede tener un diabético? Las enfermedades periodontales son más frecuentes en diabéticos. Un gran número de estudios ha demostrado que las en- fermedades periodontales son más frecuentes $y$ más avanzadas en diabéticos, tanto de tipo I como tipo 2, respecto a no diabéticos". En un trabajo de revisión de Taylor y Borgnakke, se evaluaron 17 estudios transversales realizados desde el año 2000 , y que indicaban que la diabetes afecta negativamente a la salud periodontal, lo que había sido ya sugerido por la evaluación de 48 trabajos observacionales realizados entre 1960-2000'2. Las enfermedades periodontales progresan más rápido en diabéticos. En una revisión sistemática Khader y col. sobre 23 estudios transversales o de cohortes, realizados entre 1970 y 2003, con meta-análisis de 18 de ellos, se observó que los individuos con diabetes tenían mayor grado de afectación periodontal (en cuanto a severidad), que las personas sin diabetes $^{13}$. Las enfermedades periodontales son más frecuentes $y$ progresan más rápido en diabéticos mal controlados. Los conocimientos actuales señalan que el riesgo de complicaciones asociadas a la diabetes, incluidas las enfermedades periodontales, tienen que ver con la duración de la diabetes y con su control. Así, en el estudio NHANES III (National Health and Nutrition Survey) realizado en EE.UU., los adultos con diabetes mal controlada mostraron un riesgo 2.9 más alto de tener enfermedad periodontal que aquellos que no tenían diabetes ${ }^{14}$. El mal control de la glucemia en pacientes con diabetes también ha sido asociado con un incremento del riesgo de pérdida de nivel de inserción y hueso alveolar, con el paso del tiempo ${ }^{15}$. El tratamiento de las enfermedades periodontales es tan efectivo en diabéticos como en no diabéticos. Por otra parte, el tratamiento convencional de las enfermedades periodontales, con raspado y alisado radicular, consigue una mejoría en la salud de los tejidos que rodean al diente, tanto en pacientes diabéticos como no diabéticos ${ }^{16}$. ¿Qué otros problemas bucales pueden tener un diabético? Se han encontrado otras afectaciones bucales en los individuos diabéticos, con mucha menor frecuencia y relevancia: infecciones oportunistas, como candidiasis oral, mucomicosis o ficomicosis, boca seca o xerostomía, asociada más a los tratamientos farmacológicos, síndrome de ardor bucal. Agrandamiento de las glándulas salivales. Liquen plano, aunque está discutido. Infecciones post-exodoncia. Alteraciones en 
DIVULGACIÓN

Diabetes y enfermedad periodontal, una relación de doble vía la percepción del gusto. Los adultos con diabetes mal controlada mostraron un riesgo 2.9 más alto de tener enfermedad periodontal que aquellos que no tenían diabetes. Queda claro que la salud bucal y periodontal debe ser parte integrante en el manejo de la diabetes ${ }^{17}$. En este contexto, el aumento en el conocimiento de la relación entre diabetes mellitus y enfermedad periodontal, debe llegar a los profesionales de la salud, pero también a los pacientes diabéticos. Muchas personas con diabetes son poco conscientes de las complicaciones (incluidas las bucales), asociadas con la diabetes, y reciben poca información de los profesionales que eventualmente los asisten. Por eso es necesario entrenar a los mismos para que sepan informar a sus pacientes sobre los beneficios de la buena salud oral en relación con su diabetes ${ }^{18}$. La colaboración entre profesionales de la medicina y la odontología se convierte en algo cada vez más importante. De hecho, los profesionales de la salud bucal tienen una gran oportunidad de identificar diabetes no diagnosticada o prediabetes, en pacientes odontológicos, y referir a esos pacientes a la consulta del médico o especialista ${ }^{19}$. En base a lo anterior, la Federación Internacional de Diabetes ha establecido una serie de recomendaciones para el cuidado de los pacientes diabéticos: Evaluar anualmente si el paciente cumple las recomendaciones diarias de cuidado bucal y si atiende las revisiones profesionales necesarias. Evaluar anualmente los síntomas de problema. en las encías (incluido sangrado al cepillarse o encías enrojecidas o hinchadas). Si el paciente no realiza los cuidados diarios adecuados, hay que recordarles que estos forman parte del control de la diabetes y recordar la importancia de las revisiones periódicas con el odontólogo ${ }^{20}$. Si hay sospechas de que el paciente pudiera tener problemas periodontales, se le debe recomendar acudir al odontólogo. La educación de las personas con diabetes debe incluir las implicancias de la diabetes, especialmente si está mal controlada, en la salud bucal, especialmente en la salud periodontal ${ }^{21}$. Existiría un huésped susceptible a ambas enfermedades. Intervienen mecanismos genéticos e inmunológicos. En cuanto a los primeros, tanto la diabetes como la periodontitis tienen un patrón de herencia familiar muy fuerte, existiendo en ambas una asociación entre la susceptibilidad genética y el genotipo HLA. Parece por tanto posible proponer que una combinación determinada de alelos HLA puede resultar en un huésped susceptible a ambas enfermedades. Por otro lado, ambas enfermedades pueden ser consideradas respuestas inadecuadas del sistema inmune a factores ambientales que actúan sobre un huésped predispuesto. En el caso de la periodontitis, dichos factores serían la placa bacteriana, el tabaco y el estrés. Para la DM tipo I serían los virus, micobacterias, agentes tóxicos, estrés emocional y constituyentes de las comidas, y para el tipo 2, el exceso de comida y el ejercicio ${ }^{22}$. Los profesionales de la salud bucal tienen una gran oportunidad de identificar diabetes no diagnosticada o prediabetes, en pacientes odontológicos, y referir a esos pacientes a la consulta del médico o especialista. Es necesario entrenar a los profesionales de la salud e informar a los pacientes, sobre los beneficios de la buena salud oral en relación con su diabetes ${ }^{23}$.

\section{Conclusiones}

El mecanismo biológico aceptado en la actualidad que intenta explicar el porqué los pacientes diabéticos presentan periodontitis más severas defiende, por un lado, que la acumulación de AGEs afectaría la migración y la fagocitosis tanto de polimorfonucleares como de mononucleares, resultando en el establecimiento de una flora subgingival que por maduración se irá transformando en predominantemente anaerobia gramnegativa. Esto va a desencadenar la secreción de mediadores solubles que mediarán la destrucción del tejido conectivo y la reabsorción ósea, y el establecimiento de un estado de resistencia de los tejidos frente a la insulina. Simultáneamente por otro lado, la infección periodontal también inducirá a un estado de resistencia de los tejidos frente a la insulina contribuyendo a la hiperglucemia y consecuentemente a la acumulación de AGEs. La relación que se establece entre diabetes mellitus y periodontitis, por tanto, es bidireccional resultando en una mayor severidad de la periodontitis $y$ una mayor dificultad para controlar los niveles de glucosa en sangre en pacientes diabéticos. 
REVISTA FACULTAD DE ODONTOLOGÍA

ISSN No 1668-7280- Vol. XI No 1- 2018

38

DIVULGACION

Monzón - Caramello - Acuña

\section{Referencias Bibliográficas}

I. Ardila Medina CM, Botero Zuluaga L, Guzmán Zuluaga IC. Asociación entre periodontitis crónica y altos niveles de glicemia en pacientes no diabéticos. AMC. 20|4; |8(5):507-5|8.

2. Páez González Y, Tamayo Ortiz B, Batista BoniIlo A, García Rodríguez YC Guerrero Ricardo I. Factores de riesgo de periodontopatías en pacientes adultos. CCM. 20I5; I9(2) 269-28I.

3. Taylor, G. W., Burt, B. A., Becker, M. P., Genco, R. J., Shlossman, M., Knowler, W. C. \& Pettitt, D. J. Non-insulin dependent diabetes mellitus and alveolar bone loss progression over 2 years. J Periodontol. 1998;69,76-83.

4. Faria-Almeida R, Navarro A, Bascones A. Clinical and metabolic changes after conventional treatment of type 2 diabetic patients with chronic periodontitis. J Periodontol. 2006;77,59|-8.

5. Llambés F, Arias-Herrera S, Caffesse R. Relationship between diabetes and periodontal infection. World J Diabetes. 2015; 6(7):927-935.

6. Preshaw P. Oral health awareness in adult patients with diabetes: a questionnaire study. $\mathrm{Br}$ Dent J. 201 I;21 1,274-5.

7. Teeuw, W. J., Gerdes, V. E. \& Loos, B. G. (2010) Effect of periodontal treatment on glycemic control of diabetic patients: a systematic review and meta-analysis. Diabetes Care. 2010;33,421-427.

8. Janket, S. J., Wightman, A., Baird, A. E., Van Dyke, T. E. \& Jones, J. A. (2005) Does periodontal treatment improve glycemic control in diabetic patients? A meta-analysis of intervention studies. J Dent Res. 2005;84, I I 54- I I 59.

9. Lalla, E. \& Papapanou, P. N. (20II) Diabetes mellitus and periodontitis: a tale of two common interrelated diseases. Nat Rev Endocrinol. 28.

10. Preshaw P.M., Alba A.L., Herrera D., Jepsen S., Konstantinidis A., Makrilakis K., Taylor R. Periodontitis and Diabetes: A two way relationship. Diabetologia. 2012 Jan; 55(I): 2I-3I.

II. Laplace Pérez BN, Legrá Matos SM, Fernández Laplace J, Quiñones Márquez D, Piña Suárez L, Castellanos Almestoy L. Enfermedades bucales en el adulto mayor. CCM. 20I3; I7(4):477-488.

12. Taylor GW, Borgnakke WS. (2008) Periodontal disease: associations with diabetes, glycemic control and complications. Oral Diseases. 2008; |4, | 9|-203.

13. Khader YS, Dauod AS, El-Qaderi SS, et al. Periodontal status of diabetics compared with nondiabetics: a meta-analysis. J Diabetes Complications. 2006;20,59-68.
14. Tsai, C., Hayes, C. \& Taylor, G. W. Glycemic control of type 2 diabetes and severe periodontal disease in the US adult population. Community Dent Oral Epidemiol. 2002; 30, 182-192.

15. Taylor, G. W., Burt, B. A., Becker, M. P., Genco, R. J., Shlossman, M., Knowler, W. C. \& Pettitt, D. J. (1996) Severe periodontitis and risk for poor glycemic control in patients with non-insulindependent diabetes mellitus. J Periodontol. 1996;67,1085-1093.

16. Peña Lage M, Manresa Reyes L, Rodríguez Legrá E. Enfermedad periodontal y síndrome metabólico. CCM. 20|4;|8(2):324-326.

17. Santos Tunes, R., Foss-Freitas, M. C. \& Nogueira-Filho Gda, R. (20I0) Impact of periodontitis on the diabetes-related inflammatory status. J Can Dent Assoc. 2010;76,35.

18. ADA, American Diabetes Association (20II) Diagnosis and classification of diabetes mellitus. Diabetes Care. 201 I;34 Suppl I, S62-69.

19. Mealey, B. L. \& Ocampo, G. L. Diabetes mellitus and periodontal disease. Periodontol 2000. 2007;44, I 27-I 53.

20. IDF Clinical Guidelines Task Force. IDF Guideline on oral health for people with diabetes. Brussels: International Diabetes Federation, 2009.

21. Sacks DB, Arnold M, Bakris GL, Bruns DE, Horvath $A R$, Kirkman MS, Lernmark A, Metzger BE, Nathan DM. Guidelines and recommendations for laboratory analysis in the diagnosis and management of diabetes mellitus. Diabetes Care. 20II; 34, e6I-99.

22. Wang, T. T., Chen, T. H., Wang, P. E., Lai, H., Lo, M. T., Chen, P. Y. \& Chiu, S. Y. A populationbased study on the association between type 2 diabetes and periodontal disease in 12,123 middle-aged Taiwanese (KCIS No. 21). J Clin Periodontol. 2009;36, 372-379.

23. Wild, S., Roglic, G., Green, A., Sicree, R. \& King, $\mathrm{H}$. Global prevalence of diabetes: estimates for the year 2000 and projections for 2030. Diabetes Care. 2004;27, 1047-1053. A populationbased study on the association between type 2 diabetes and periodontal disease in 12,123 middle-aged Taiwanese (KCIS No. 2I). J Clin Periodontol $36,372-379$. 